\title{
O CONSUMO DO ÁLCOOL E AS DOENÇAS CARDIOVASCULARES - UMA ANÁLISE SOB O OLHAR DA ENFERMAGEM
}

\author{
The consumption of alcohol and the cardiovascular \\ diseases - an analysis under the nursing view \\ El consumo de alcohol y las enfermedades \\ cardiovasculares - un análisis desde la óptica de la enfermería
}

Marluci Andrade Conceição Stipp
Luana Santos de Assis ${ }^{4}$

\author{
Joséte Luzia Leite ${ }^{2}$ \\ Michel Pires de Andrade ${ }^{5}$
}

\author{
Natália Machado da Cunha ${ }^{3}$ \\ Ricardo Duarte Simões ${ }^{6}$
}

\section{Resumo}

Estudo descritivo, exploratório e quantitativo, que apresenta uma análise parcial dos dados, cujos objetivos foram estimar a freqüência do uso de bebida alcoólica numa clientela com hipertensão arterial atendida no ambulatório de um hospital-escola do Município do Rio de Janeiro e discutir a relação do uso do álcool com as doenças cardiovasculares. A coleta de informações ocorreu num hospital-escola do Município do Rio de Janeiro. Foi utilizado um questionário aplicado oralmente a 73 clientes. Encontramos 59,9\% de hipertensos, e, destes, 91,30\% fazem uso de alguma medicação anti-hipertensiva e 74,36\% referem não ingerir bebida alcoólica. Do total de entrevistados, 84,93\% eram do sexo feminino. Foi observado na literatura que o uso moderado do álcool aponta uma proteção contra doença arterial coronariana. 0 estudo mostrou um controle da freqüência da ingesta alcoólica, o que pode estar relacionado ao tempo de acompanhamento ambulatorial desta clientela. Foi vista uma participação efetiva de enfermeiras, alunos e docentes de Enfermagem na assistência ambulatorial da referida instituição.

Palavras-chave: Enfermagem. Bebidas Alcoólicas. Doenças Cardiovasculares.

\begin{abstract}
Descriptive, exploratory and quantitative study that in the moment presents a partial data analysis with the purpose of to consider the frequency of the alcoholic beverage use in a clientele with Arterial Hypertension attended in an ambulatory of a School Hospital of the Rio de Janeiro City and to discuss the relation between the alcohol use and the cardiovascular disease. The information collect was made in a School Hospital of the Rio de Janeiro City. It was used an orally applied questionnaire in 73 clients. We found $59.9 \%$ of hypertensive clients, from which $91.30 \%$ use some kind of medication against Hypertension and $74.36 \%$ refer to don't use alcoholic beverage. From the total of interviewed people $84.93 \%$ was from the feminine gender. It was observed in the literature that the moderate use of alcohol points to a protection against coronaries arterial disease. The study showed a control of the frequency of the alcohol ingestion which can be related to the time of ambulatory accompaniment of these clientele. It was examined an effective participation of nurses, students and teachers of nursing in the ambulatory assistance of the referred institution.
\end{abstract}

Keywords:

Nursing. Alcoholic Beverages. Cardiovascular Diseases.

\section{Resumen}

Estudio descriptivo, exploratorio, cuantitativo y en el momento presenta un análisis parcial de los datos cuyo objetivo fue estimar la frecuencia del uso de bebida alcohólica en una clientela con Hipertensión Arterial atendida en el ambulatorio de un Hospital Escuela de la Ciudad del Rio de Janeiro y discutir la relación del uso del alcohol con las enfermedades cardiovasculares. La colecta de informaciones ocurrió en un Hospital Escuela de la Ciudad del Rio de Janeiro. Fue usado un cuestionario aplicado oralmente a 73 clientes. Encontramos 59,9\% de hipertensos, y de estos $91,30 \%$ hacen uso de alguna medicación antihipertensiva y $74,36 \%$ mencionan a no ingerir bebida alcohólica. Del total de entrevistados $84,93 \%$ eran del sexo femenino. Fue observado en la literatura que el uso moderado de alcohol apunta una protección contra enfermedad arterial coronariana. El estudio muestra un control de la frecuencia de ingestión alcohólica lo cual puede estar relacionado al tiempo de acompañamiento de esta clientelaen el ambulatorio. Fue visto una participación efectiva de enfermeras, alumnos y docentes de enfermería en la ayuda en el ambulatorio de la referida institución. 


\section{INTRODUÇÃO}

Os fatores de risco têm sido estudados como verdadeiros agentes causais das doenças não transmissíveis, em especial as doenças cardiovasculares, o câncer e o diabetes mellitus. No caso das doenças cardiovasculares, foco de nosso estudo, importantes características comportamentais ligadas ao estilo de vida são fatores determinantes que auxiliam no tratamento dessa doença.

Geralmente, a evolução clínica para as doenças cardiovasculares é lenta, relacionada a uma multiplicidade de fatores de risco, que, quando não tratada, acarreta complicações sérias para saúde, sendo algumas delas de caráter permanente. A literatura ${ }^{1}$ destaca o importante ônus gerado por estas doenças à sociedade, principalmente relacionado aos agravos das doenças cerebrovasculares, doença arterial coronária, insuficiências cardíaca e renal crônicas e doença vascular de extremidades.

A associação entre o consumo de bebidas alcoólicas e as doenças cardiovasculares é uma questão merecedora de especial atenção, uma vez que a investigação sobre esse consumo estará ligada ao rigor metodológico de cada pesquisa nos diversos estudos sobre o tema. Muitos são os questionamentos que se fazem em torno da temática ser ou não alcoolista, até que ponto o uso do álcool é benéfico para o indivíduo? Quais os prejuízos acarretados para o indivíduo, família e sociedade?

Partindo desses questionamentos, resolvemos focalizar nosso estudo discutindo a relação do álcool com as doenças cardiovasculares, em específico a Hipertensão Arterial, pela sua forte relação com a doença coronariana obstrutiva. Os objetivos deste estudo foram estimar a frequêencia do uso de bebida alcoólica numa clientela com hipertensão arterial atendida no ambulatório de um hospital-escola do Município do Rio de Janeiro e discutir a relação do uso do álcool com as doenças cardiovasculares.

Dentre as doenças de maior relevância epidemiológica, destacamse as doenças cardiovasculares, e, para tal, os fatores de risco cardiovasculares mais importantes têm sido atribuídos à hipertensão arterial, diabetes mellitus, hipercolesterolemia e obesidade.

Alguns principais fatores de risco são conhecidos e comprovados na literatura nacional e internacional, entre eles os não modificáveis, como a idade, sexo e história familiar. Entre os modificáveis temos: hipertensão arterial, diabetes mellitus, dislipidemias, obesidade, sedentarismo, tabagismo, dentre outros ${ }^{2,3}$.

Os clientes portadores de cardiopatias devem ser orientados quanto à manutenção ou mudança de um estilo de vida adequado para a promoção de sua saúde, e promover a sua perda de peso (caso esteja acima do padrão), restringindo o sódio de sua dieta e inserindo frutas e vegetais ${ }^{4}$; além disso, devem ser encorajados a modificar outros hábitos, tais como a redução da ingesta alcoólica.

0 álcool é uma substância tóxica que pode contribuir para ocorrência de algumas doenças como: hipertensão arterial, hipertrigliceridemia, diabetes não-insulinodependente, câncer, hepatopatia, encefalopatia, pancreatite, problemas psicossociais e comportamentais 5 . Seu uso crônico e não moderado acarreta prejuízo no convívio social e pode ser visto como uma importante patologia social. É considerado uma droga lícita, sem restrições para seu consumo, o que muitas vezes leva homens e mulheres a seu uso abusivo.

0 consumo do álcool afeta diferentes grupos étnicos, não depende de idade, classe social, econômica e gênero. De acordo com o Sétimo Relatório da Reunião Nacional do Comitê sobre Prevenção, Detecção, Avaliação e Tratamento da Hipertensão Arterial (The JNC 7 Report) ${ }^{6}$, a ingesta moderada não deve ultrapassar mais de 2 drinks por dia, o que corresponde a não mais de $30 \mathrm{ml}$ de etanol, ou $720 \mathrm{ml}$ de cerveja, ou $300 \mathrm{ml}$ de vinho ou $60 \mathrm{ml}$ de uísque $100 \%$ puro. Para os homens e pessoas com menor densidade corporal, como no caso das mulheres e indivíduos mais magros, estes seriam aconselhados a não ultrapassar $15 \mathrm{ml}$ de etanol diários.

Entendemos que esta ingesta moderada a que se refere a literatura necessita de uma avaliação do contexto social a que esse indivíduo está inserido, bem como da sua patologia de base e da presença de outras doenças associadas. A abstenção ou restrição da ingesta de álcool são medidas eficazes na redução arterial, dependendo de cada caso clínico avaliado individualmente.

\section{MÉTODOS E TÉCNICAS}

Estudo descritivo, exploratório, de natureza quantitativa, com uma coleta sistemática de informações e análise utilizando procedimentos estatísticos ${ }^{7}$. Apresenta uma análise parcial dos resultados oriunda do Projeto Integrado de Pesquisa intitulado "Prevalência das Doenças Cardiovasculares na Clientela Atendida em um Hospital-Escola do Município do Rio de Janeiro". Este projeto está em andamento e é parte integrante de um projeto "guarda-chuva", desenvolvido pelo grupo de pesquisa intitulado "Enfermagem Cardiovascular: Aspectos gerenciais e de educação em saúde", registrado no diretório de grupo de pesquisa do Conselho Nacional de Desenvolvimento Científico e Tecnológico (CNPq).

A população foi constituída de clientes atendidos ambulatorialmente no Hospital Escola São Francisco de Assis da Universidade Federal do Rio de Janeiro. Os critérios para elegibilidade dos indivíduos do estudo foram: clientes de ambos os sexos, acima de 18 anos, em condições clínicas de se comunicar, que buscavam atendimento ambulatorial nas diversas especialidades clínicas do hospital e que aceitaram mediante apresentação e assinatura do Termo de Consentimento Livre e Esclarecido.

Foi assegurado aos participantes sigilo quanto às informações prestadas, de acordo com a Resolução n 196/06, do Conselho Nacional de Saúde. A pesquisa foi aprovada pelo Comitê de Ética e Pesquisa da Escola de Enfermagem Anna Nery (EEAN) e Hospital Escola São Francisco de Assis (HESFA) da Universidade Federal do Rio de Janeiro (UFRJ), protocolo $n^{\circ}$ 044/77.

0 instrumento para coleta de dados foi um questionário aplicado oralmente com perguntas sobre o perfil de risco para as doenças cardiovasculares: idade, sexo, história familiar, obesidade, hipertensão arterial, diabetes mellitus, dislipidemia, tabagismo, etilismo, sedentarismo, estresse, menopausa, reposição hormonal. Para realização deste estudo foram analisados os dados coletados no período da manhã e da tarde, de junho a agosto de 2007. 
Antes da aplicação do instrumento, foi realizado um treinamento com a pesquisadora responsável e os alunos de Iniciação Cientifica inseridos no projeto, para que os mesmos aplicassem o questionário de forma uniformizada, garantindo a correta coleta das informações. Foi realizado um préteste para assegurar validade e precisão do instrumento, conforme preconizado por Gil ${ }^{8}$.

Os dados foram coletados por questionários aplicados por entrevista pelos membros da equipe do projeto na sala de espera do ambulatório. Os clientes foram escolhidos de forma aleatória enquanto aguardavam a consulta dos profissionais de saúde. Os resultados foram tabulados e organizados, utilizando o método de estatística descritiva, de freqüência simples e percentual.

\section{APRESENTAÇÃO DOS RESULTADOS}

Participaram deste estudo até o momento 73 entrevistados, sendo $84,93 \%$ do sexo feminino e $15,7 \%$ do sexo masculino. Deles, 58,90\% apresentavam vida conjugal, $60,27 \%$ possuíam renda familiar entre um a três salários mínimos, e, quanto à escolaridade, $52,5 \%$ possuíam o ensino fundamental incompleto. A maioria se concentrou na faixa etária entre 41 e 60 anos, perfazendo um total de 57,53\%. Com relação ao acesso a uma Unidade Básica de Saúde, $83,56 \%$ dos entrevistados relataram ter um fácil acesso ao atendimento de saúde.

Dos entrevistados, 59,9\% eram hipertensos, e, destes com diagnóstico de hipertensão, 91,30\% faziam uso de alguma medicação anti-hipertensiva e $74,36 \%$ referiram não ingerir bebida alcoólica. Dentre os usuários (hipertensos e não hipertensos), 28,77\% relataram baixa freqüência no consumo de bebida alcoólica, variando de uma a duas vezes por semana.

É importante relatar que, com relação ao diabetes mellitus, apesar de sua alta prevalência na população, até o momento apenas uma pessoa apresentava diabetes e sete tinham hipertensão e diabetes associado, correspondendo a 1,52\% e $9,59 \%$ respectivamente.

No Brasil, a estimativa de prevalência da hipertensão arterial varia de $22,3 \%$ a $44 \%$ de acordo com a área estudada ${ }^{9}$, e há uma predominância das maiores taxas principalmente nos trabalhos mais recentes ${ }^{10}$.

No que diz respeito ao tempo de acompanhamento ambulatorial dos entrevistados, foi visto que $34,78 \%$ possuem o diagnóstico de hipertensão arterial num período que varia de 6 a 10 anos, seguido de $21,74 \%$ no período de um a cinco anos; $19,56 \%$ têm um período de mais de 20 anos com hipertensão arterial sabidamente diagnosticada; $13,4 \%$, um período de 11 a 20 anos; $6,52 \%$ realizam acompanhamento com menos de um ano e apenas 4,35\% não souberam informar.

\section{DISCUSSÃO DOS DADOS}

0 uso do álcool é lícito em nossa cultura, e, apesar dessa característica importante em nosso meio, encontramos uma alta freqüência de não-usuários de bebida alcoólica em nossa pesquisa. A referida instituição atende em média 2.266 pacientes por mês, no entanto, por nosso estudo estar ainda em fase inicial, é possível que a freqüência do uso de bebida alcoólica se modifique com o caminhar da coleta de dados.

Com relação à hipertensão arterial, a restrição da ingestão de álcool é uma medida eficaz na redução da pressão arterial, assim como a redução do estresse, eliminação do tabagismo, prática regular de atividade física, perda de peso, dentre outros fatores associados ao estilo de vida das pessoas.

Encontramos dentre os poucos usuários uma baixa freqüência do uso de bebida alcoólica, variando de uma a duas vezes por semana. Amoedo et al. ${ }^{11}$ informam que a diminuição do uso de álcool diminui a pressão sistólica aproximadamente $2-4 \mathrm{mmHg}$. A ocorrência de hipertensão secundária ao consumo de álcool varia de $5 \%$ a $11 \%$, em estudos com diferentes populações, indicando a idéia de uma causa potencialmente tratável de hipertensão.

No estudo de Stranges et al..$^{12}$, foi observado que o risco de hipertensão arterial associado à ingestão recente de duas ou mais doses de etanol/dia é maior que o dobro, comparado a abstêmicos. Demonstrou, ainda, uma forte relação independente de risco para álcool ingerido fora do horário das refeições; os que consumiam álcool fora das refeições tiveram maior risco de hipertensão arterial.

A graduação de ingesta alcoólica feita pelo National Center of Health Statistics é a de consumidor infreqüente e consumidor regular. 0 primeiro ingere álcool até duas vezes por semana e subdivide-se em leve (uma a duas doses por dia); moderado (três a quatro doses por dia) e pesado (cinco ou mais doses por dia). 0 consumidor regular é aquele que bebe mais que duas vezes por semana e também pode ser considerado leve, moderado e pesado ${ }^{11}$.

0 controle da freqüência da ingesta alcoólica entre os participantes desse estudo também pode estar relacionado ao fato de ser a grande parte dos entrevistados do sexo feminino e ao tempo de acompanhamento ambulatorial destes clientes, pois a maioria freqüenta 0 ambulatório há mais de 5 anos. 0 fato de a grande maioria ser de mulheres é um fator positivo quando se refere ao acompanhamento ambulatorial, pois o mesmo, devido ao controle preventivo do câncer de colo uterino e de mama, que se fazem anualmente nos consultórios médicos ou de enfermagem, possibilita um controle maior de outras condições de saúde crônicas ou agudas pelos profissionais de saúde.

Vale destacar que a instituição em que se realiza o estudo possui um Centro de Estudos de Prevenção e Reabilitação do Alcoolismo (CEPRAL) com participação de uma equipe multiprofissional incluindo docentes de enfermagem. A participação de enfermeiras, docentes e alunos de enfermagem nas atividades do HESFA é de suma importância, principalmente no que tange à pesquisa e a atividades de extensão universitária.

0 HESFA dispõe ainda de tratamentos alternativos, como ambulatórios de homeopatia e acumpultura, além de contar com assistência primária e secundária nas diversas especialidades clínicas, bem como consultas de enfermagem nas áreas de ginecologia primária (preventivo de colo uterino e mama), doenças sexualmente transmissível e pré-natal, de acordo com os protocolos do Ministério da Saúde.

Atividades de educação em saúde nas salas de espera também são realizadas pelos acadêmicos de enfermagem, servindo como uma atividade de intervenção em saúde, 
proporcionado informação à clientela sobre os diversos assuntos, dentre eles os fatores de risco cardiovasculares.

Com relação às doenças cardiovasculares e o uso do álcool, estudos apontam uma proteção contra doença arterial coronariana. Porém este aspecto benéfico se faz com o consumo de quantidades pequenas ou moderadas, como a elevação da fração HDL do colesteroli3 ${ }^{13}$ Indivíduos com hipertrigliceridemia, diabetes e hepatopatia devem evitar o álcool ${ }^{14}$.

Uma metanálise ${ }^{15}$ sobre o uso moderado de consumo de álcool e o risco de desenvolver doença coronariana mostrou que, comparado com a abstenção, o consumo de até aproximadamente $30 \mathrm{~g} /$ dia (aproximadamente duas doses diárias de bebida alcoólica), é casualmente relacionado com $20 \%$ a $45 \%$ de redução de desenvolver doença coronariana. Outros estudos ${ }^{16}$ relatam que este consumo moderado não está relacionado a um tipo especial de bebida alcoólica, embora o vinho tinto seja visto como importante no senso comum de países como a França.

Outro dado merecedor de atenção foi o fácil acesso dos clientes entrevistados a uma unidade básica de saúde. Uma característica da Unidade Ambulatorial do HESFA não é apenas atender a uma área programática, mas a uma demanda espontânea. São clientes oriundos de diversos locais do Município do Rio de Janeiro, de outras instituições e dos programas desenvolvidos no próprio HESFA.

Dessa forma, apesar de o hospital contar apenas com recursos do Sistema Único de Saúde, a instituição preserva um estilo gerencial que garante o atendimento de uma demanda espontânea e a continuidade das atividades assistenciais primária e secundária, firmando-se cada vez mais como um Hospital-Dia.

\section{CONSIDERAÇÕES FINAIS}

Percebemos que o uso do álcool está relacionado ao hábito de vida de cada um, assim como uma importante variável passível de ser controlada pelo próprio indivíduo. Ainda não existe um modelo único a seguir para o planejamento de cuidados de enfermagem para esta clientela específica.

\section{Referências}

1. Toledo MM, Rodrigues SC, Chiesa AM. Educação em saúde no enfrentamento da hipertensão arterial: uma nova ótica para um velho problema. Texto\&Contexto Enferm 2007 abr/jun; 16(2):233-38.

2. Santoro DC, Stipp MAC, Leite JL, Stipp CA. 0 cliente com doença coronariana e os fatores de riscos. In: Figueiredo MAF, Stipp MAC, Leite, J,organizadores. Cardiopatias: avaliação e intervenção em enfermagem. São Paulo(SP): Yendis; 2006. p. 81-103.

3. Oliveira GMM, Klein CH, Silva NAS. Mortalidade por doenças isquêmicas do coração, doenças cerebrovasculares e causas mal definidas nas regiões de saúde do estado do Rio de Janeiro, no período de 1980 a 2000. Rev SOCERJ 2005 jan/fev; 13-29

4. Stipp MAC, Aguiar DF. Produção científica do cuidado ambulatorial de enfermagem à clientela portadora de hipertensão arterial Online Braz J Nurs 20076:1-10. Disponível em: http://www.uff.br/objnursing/index.php/nursing.
Estudos brasileiros de Enfermagem ainda são escassos sobre a temática das doenças cardiovasculares e o uso de álcool. 0 presente estudo, mesmo que ainda seja uma análise parcial dos dados, mostrou uma clientela pouco usuária de bebida alcoólica, apesar de ainda não analisarmos quantitativamente a ingestão de álcool.

Diante dos resultados obtidos, por ser a maior parte da clientela entrevistada do sexo feminino, este fator pode justificar a baixa freqüência do uso de álcool, mesmo sabendo que a incidência dos fatores de risco que antes predominavam numa clientela do sexo masculino, como no caso do tabagismo, estresse e etilismo, atualmente também é bastante observada entre as mulheres.

Outro fator importante também relacionado à questão de gênero é que trabalhamos com uma clientela feminina hipertensa que procura uma assistência ambulatorial e recebe atendimento nas diversas especialidades, entre elas intervenções educativas nas salas de espera, com foco principalmente nos programas de prevenção de doenças crônicas degenerativas, entre outras.

No estudo de Minto et al. ${ }^{17}$, foi observado que, entre estratégias de prevenção e tratamento de problemas relacionados ao álcool, as intervenções breves têm sido relatadas como bastante eficazes, principalmente quando realizadas em condições especiais de pesquisa, embora pouco se conheça sobre o desempenho dessas práticas em situações usuais de atendimento na rotina dos serviços de saúde.

A mulher, como já é sabido, tem um histórico de freqüentar mais os serviços de saúde; além do mais, grande parte faz uso de medicação anti-hipertensiva, o que pode propiciar um maior controle no hábito de ingestão de bebida alcoólica.

0 uso do álcool é considerado um dos maiores problemas de saúde pública que pode ser combatido através de uma intervenção por qualquer profissional da saúde que esteja engajado em um programa de promoção a saúde, não o subestimando como fator de risco, realizando uma investigação correta e registrando-o de maneira rotineira.

5. Bloch K V. Fatores de risco cardiovasculares e para o diabetes mellitus. In: Lessa I, organizador. 0 adulto brasileiro e as doenças da modernidade. Epidemiologia das doenças crônica não transmissíveis. Rio de Janeiro(RJ): /Hucitec; 1998. p.43-72.

6. Chobaniam AV, Bahris GL, Black HR, Cushman WC, Green LA, Izzo Junior. JL, et al. $7^{\circ}$ Report of the Joint National Committee on Prevention, Detection, Evaluation, and Treatment of High Blood Pressure (The JNC 7 Report). JAMA 2003; 289(19): 2560-572

7. LoBiondo-Wood G, Haber J. Pesquisa em enfermagem: métodos, avaliação crítica e utilização. $4^{\mathrm{a}}$ ed. Rio de Janeiro(RJ): Guanabara Koogan; 2001.

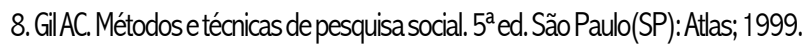

9. Sociedade Brasileira de Cardiologia. Sociedade Brasileira de Hipertensão. Sociedade Brasileira de Nefrologia. IV Diretrizes Brasileiras de Hipertensão Arterial. Arq Bras Cardiol 2004 ( 4 supl.): 1 - 40

10. Souza ARA Costa A, Nakamura D, Mocheti LN, Stevanato Filho PR, Ovando LA. Um estudo sobre hipertensão arterial sistêmica na cidade de Campo Grande. Arq Bras Cardiol 2007; 88(4): 441-46. 
11. Amoedo C, Passarelli Junior O, Borelli FAO, Souza MG. Tratamento não medicamentoso da hipertensão arterial. In: Nobre F, Serrano Junior CV, organizador. Tratado de cardiologia SOCESP. São Paulo(SP): Manole; 2005. p.453-63.

12. Stranges S, Wu T, Dorn J M, Freudenheim J L, Muti P, Farinaro E, et al. Realtionship of alcohol drink pattern to risk of hypertension: a population based-study. Hypertension 2004; 44 (6): 813-19.

13. Avezum A, Guimarães HP, Piegas LS. Fatores de risco associados com infar to agudo do miocárdio na região metropolitana de São Paulo e no Brasil. In: Nobre F, Serrano Junior CV, organizadores. Tratado de cardiologia SOCESP. São Paulo(SP): Manole; 2005. p.22-33.

\section{André C. Manual do AVC. Rio de Janeiro(RJ): Revinter; 1999}

15. Rimm EB, Williams P, Fosher K, Criqui M, Stampfer MJ. Moderate alcohol intake and lower risk of coronary heart disease: meta-analysis of effects on lipids and haemostatic factors. Br Med J 1999; 319:1523-528.

16. Goldfinger TM. Beyond the french paradox: the impact of moderate beverage alcohol and wine consumption in the prevention of cardiovascular disease. Cardiol Clin 2003; 21: 449 - 57.

17. Minto EC, Corradi-Webster CM, Gorayeb R, Laprega MR, Furtado EF. Intervenções breves para o uso abusivo do álcool em atenção primária. Epidemiol Serv Saude 2007; 16 (3): 207-20. 